\title{
Joint CFO and Channel Estimation for CP-OFDM Modulated Two-Way Relay Networks
}

\author{
Gongpu Wang ${ }^{\dagger}$, Feifei Gao ${ }^{\ddagger}$, Yik-Chung Wu*, and Chintha Tellambura ${ }^{\dagger}$ \\ ${ }^{\dagger}$ Department of Electrical and Computer Engineering, University of Alberta, Edmonton, Canada \\ Email: \{gongpu, chintha\}@ece.ualberta.ca \\ ${ }^{\ddagger}$ School of Engineering and Science, Jacobs University, Bremen, Germany \\ Email: feifeigao@ieee.org \\ *Department of Electrical and Electronic Engineering, University of Hong Kong, Hong Kong \\ Email: ycwu@eee.hku.hk
}

\begin{abstract}
In this paper, we study the problem of joint carrier frequency offset (CFO) and channel estimation for amplify-andforward (AF) two-way relay network (TWRN) that comprises two source terminals and one relay node. Both the system design and the estimation problem become more challenging when CFO is non-zero in a frequency-selective environment, as compared to the conventional point-to-point communication systems. By introducing some redundancy, we propose a cyclic prefix (CP) based OFDM modulation for TWRN that is capable of maintaining the advantage of using multi-carrier transmission and at the same time facilitates the system initialization, e.g., synchronization and channel estimation. We then apply a least square (LS) approach to solve the estimation problem. The approximated Cramér-Rao Bound (CRB) has been derived as the performance benchmark of the proposed estimator. Finally, simulations are provided to corroborate the theoretical studies.
\end{abstract}

\section{INTRODUCTION}

Research on relay networks has become popular since the pioneer work [1] when Laneman et al. developed lowcomplexity cooperative diversity strategies and characterized the system performance in terms of outage probabilities. In [1], data streams flow unidirectionally from the source to the relay and then to the destination. Such derived network structure is known as the one-way relay network (OWRN). Since most communication systems are bidirectional, it is also necessary to consider the situation when the source node and the destination node exchange their roles. In fact, the signal collision at the relay can be perfectly resolved at both source terminals from a "network coding"-like manner [2]. This newly structured relay network is known as the two-way relay network (TWRN).

It has been reported in [3] that the overall communication rate between two source terminals in TWRN is approximately twice of that achieved in OWRN, making TWRN particularly attractive to any bidirectional system. Following this guideline, the capacity analysis and the achievable rate region for amplify-and-forward (AF) and decode-and-forward (DF) based TWRN were explored in [4], [5]. In [6] the optimal mapping function at the relay node that minimizes the transmission bit-error rate (BER) was proposed while in [7], the distributed space-time code (STC) was designed for both
$\mathrm{AF}$ and DF TWRN. Moreover, the optimal beamforming at the multi-antenna relay that maximizes the capacity of AF-based TWRN was developed in [8] and the suboptimal resource allocation in an orthogonal frequency division multiplexing (OFDM) based TWRN was derived in [9].

However, most existing works [3]- [9] assumed perfect synchronization and channel state information (CSI) at the relay node and/or the source terminals. Although the traditional methods can be applied to DF based TWRN, it is necessary to re-visit these two issues for AF based TWRN where the mixture between the first and the second transmission phases makes the estimation a non-trivial problem.

Our earlier study of joint carrier frequency offset (CFO) and channel estimation in TWRN [10] considered the simplest form of relay that acts as a repeater in TWRN. In this paper, we take a step further by looking into the OFDM modulated TWRN. By introducing some redundancy, we adapt the cyclic prefix (CP) based OFDM modulation scheme to TWRN that maintains the carrier orthogonality and facilitates the joint estimation process. The resultant mathematical model is different from the traditional one [11] in that the CFO only accompanies part of the signal components. We then propose a least square (LS) estimation method and derive its approximated Cramér-Rao Bound (CRB). The simulation results are later provided to verify our theoretical studies.

\section{Problem Formulation}

\section{A. System Model}

Consider a TWRN with two source nodes $\mathbb{T}_{1}$ and $\mathbb{T}_{2}$, and one relay node $\mathbb{R}$ shown in Fig. 1. Each node has only one antenna that cannot transmit and receive simultaneously. The baseband channel between $\mathbb{T}_{i}$ and $\mathbb{R}$ is denoted by

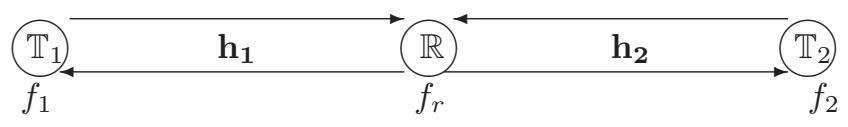

Fig. 1. System configuration for a three-node TWRN. 


$$
\begin{aligned}
\mathbf{r}_{c p} & =\sum_{i=1}^{2} e^{\jmath 2 \pi\left(f_{i}-f_{r}\right) L T_{s}} \boldsymbol{\Gamma}^{(N+L)}\left[f_{i}-f_{r}\right] \mathbf{H}_{c v}^{(N+L)}\left[\mathbf{h}_{i}\right] \mathbf{T}_{c p}^{(2 L)} \mathbf{s}_{i}+\mathbf{n}_{r} \\
& =\sum_{i=1}^{2} e^{\jmath 2 \pi\left(f_{i}-f_{r}\right) L T_{s}} \boldsymbol{\Gamma}^{(N+L)}\left[f_{i}-f_{r}\right] \mathbf{T}_{c p}^{(L)} \mathbf{H}_{c p}^{(N)}\left[\mathbf{h}_{i}\right] \mathbf{s}_{i}+\mathbf{n}_{r}
\end{aligned}
$$

$\mathbf{h}_{i}=\left[h_{i, 0}, h_{i, 1}, \ldots, h_{i, L}\right]^{T}$, where $L$ represents the order of the corresponding channel. ${ }^{1}$ The elements in $\mathbf{h}_{i}$ are assumed to be zero-mean circularly symmetric complex Gaussian (CSCG) random variables and are independent from one another. In particular, the variance of the $l$ th elements in $\mathbf{h}_{i}$ are denoted by $\sigma_{i, l}^{2}$. In this paper, we will adopt the time-division-duplexing (TDD) assumption as [2]- [9]. Then the channels can be considered reciprocal such that the channel from $\mathbb{R}$ to $\mathbb{T}_{i}$ is still represented by $\mathbf{h}_{i}$. Nonetheless, the proposed strategies can be straightforwardly extended to the more general situation when channels are non-reciprocal. The average transmission powers of $\mathbb{T}_{1}, \mathbb{T}_{2}$, and $\mathbb{R}$ are denoted as $P_{1}, P_{2}$, and $P_{r}$, respectively. And the carrier frequencies of $\mathbb{T}_{1}, \mathbb{T}_{2}$, and $\mathbb{R}$ are denoted as $f_{1}, f_{2}$, and $f_{r}$, respectively.

\section{B. Existing OFDM Modulation for TWRN}

In [12], the OFDM modulation has been adapted to $\mathrm{AF}$ TWRN for perfect synchronization scenario. The normal OFDM modulation is applied at two source terminals in the first phase, while the relay needs to remove the $\mathrm{CP}$ and add a new CP to yield the circular convolution between the channels of the first and the second phases. By doing this, the received signal is the circular convolution among the signal block and both channels, which keeps the orthogonality among different carriers.

Unfortunately, if there exist non-zero CFO among $\mathbb{T}_{1}$, $\mathbb{T}_{2}$, and $\mathbb{R}$, the circular-convolution property will be broken since the inter-carrier interference (ICI) resides in both the transmission phases. In this case, a more judicious adaption of the conventional OFDM modulation to TWRN should be designed.

\section{OFDM MODULATION WITH FREQUENCY SYNCHRONIZATION ERRORS}

\section{A. OFDM Modulation at Terminals}

Each terminal $\mathbb{T}_{i}$ first obtains the time domain OFDM signal $\mathbf{s}_{i}$ from its frequency domain information block $\tilde{\mathbf{s}}_{i}$ via the normalized IDFT approach. Suppose that each block contain $N$ symbols. We propose that $\mathbb{T}_{i}$ adds $\mathrm{CP}$ of length $2 L$ in the front of $\tilde{\mathbf{s}}_{i}$. This implicitly requires $N \geq 2 L$ which is nevertheless satisfied by most OFDM systems.

Define

$$
\mathbf{T}_{c p}^{(P)}=\left[\begin{array}{cc}
\mathbf{0} & \mathbf{I}_{P} \\
- & -- \\
\multicolumn{2}{c}{\mathbf{I}_{N}}
\end{array}\right]
$$

\footnotetext{
${ }^{1}$ We assume the same channel length of $\mathbf{h}_{i}$ 's for notation simplicity. The rest of the discussions can be straightforwardly extended to the more general case.
}

for any $P \leq N$. The baseband signal sent out from $\mathbb{T}_{i}$ is mathematically expressed as $\mathbf{T}_{c p}^{(2 L)} \mathbf{s}_{i}$, which will then be upconverted to passband signal by $e^{j 2 \pi f_{i} t}$. $^{2}$

\section{B. Relay Processing}

Relay $\mathbb{R}$ first down-converts the passband signal by $e^{-\jmath 2 \pi f_{r} t}$, which gives signal block of length $N+2 L$. However, $\mathbb{R}$ only removes the first $L$ symbol in the block. Define the convolution matrix

$$
\left.\mathbf{H}_{c v}^{(K)}[\mathbf{x}]=\left[\begin{array}{ccccc}
x_{P} & \ldots & x_{0} & \ldots & 0 \\
\vdots & \ddots & \ddots & \ddots & \vdots \\
0 & \ldots & x_{P} & \ldots & x_{0}
\end{array}\right]\right\} K \text { rows }
$$

for $\mathbf{x}=\left[x_{0}, x_{1}, \ldots, x_{P}\right]^{T}$. Also define $\boldsymbol{\Gamma}^{(N)}[f]=$ $\operatorname{diag}\left\{1, e^{j 2 \pi f T_{s}}, \ldots, e^{j 2 \pi(N-1) f T_{s}}\right\}$. The remaining signal of length $N+L$ is shown in (3) on the top of this page where the properties

$$
\begin{aligned}
\mathbf{H}_{c v}^{(N+L)}\left[\mathbf{h}_{i}\right] \mathbf{T}_{c p}^{(2 L)} & =\mathbf{T}_{c p}^{(L)} \mathbf{H}_{c v}^{(N)}\left[\mathbf{h}_{i}\right] \mathbf{T}_{c p}^{(L)} \\
\mathbf{H}_{c v}^{(N)}\left[\mathbf{h}_{i}\right] \mathbf{T}_{c p}^{(L)} & =\mathbf{H}_{c p}^{(N)}\left[\mathbf{h}_{i}\right]
\end{aligned}
$$

are used, and $\mathbf{H}_{c p}^{(N)}\left[\mathbf{h}_{i}\right]$ is the $N \times N$ circulant matrix with the first column $\left[\mathbf{h}_{i}^{T}, \mathbf{0}_{1 \times(N-L-1)}^{T}\right]^{T}$.

To keep the power constraint from a long term viewpoint, $\mathbb{R}$ scales $\mathbf{r}$ by a factor

$$
\alpha_{c p}=\sqrt{\frac{P_{r}}{\mathrm{E}\left\{\left\|\mathbf{r}_{c p}\right\|^{2}\right\}}}=\sqrt{\frac{P_{r}}{\sum_{i=1}^{2} \sum_{l=0}^{L} \sigma_{i, l}^{2} P_{i}+\sigma_{n}^{2}}},
$$

where the property that

$$
\left(\mathbf{T}_{c p}^{(L)}\right)^{H} \mathbf{T}_{c p}^{(L)}=\left[\begin{array}{cc}
\mathbf{I}_{N-L} & \mathbf{0} \\
\mathbf{0} & 2 \mathbf{I}_{L}
\end{array}\right]
$$

is used during the computation. Relay $\mathbb{R}$ then up-converts the baseband signal $\alpha_{c p} \mathbf{r}_{c p}$ by $e^{j 2 \pi f_{r} t}$ and broadcasts it to both terminals.

\section{Signal Reformulation at Terminals}

Due to symmetry, we only illustrate the process at $\mathbb{T}_{1}$. After down-converting the passband signal by $e^{-\jmath 2 \pi f_{1} t}, \mathbb{T}_{1}$ obtains the baseband block of length $N+L$. It then removes the first $L$ elements and the remaining signal is written in (8), which

\footnotetext{
${ }^{2}$ Note that the oscillator may have a initial phase but it is omitted for brevity since the constant phase can be absorbed into the channel effects.
} 


$$
\begin{aligned}
& \mathbf{y}_{c p}=\alpha_{c p} e^{j 2 \pi\left(f_{r}-f_{1}\right) L T_{s}} \boldsymbol{\Gamma}^{(N)}\left[f_{r}-f_{1}\right] \mathbf{H}_{c v}^{(N)}\left[\mathbf{h}_{1}\right] \mathbf{r}_{c p} \\
&=\alpha_{c p} \boldsymbol{\Gamma}^{(N)}\left[f_{r}-f_{1}\right] \mathbf{H}_{c v}^{(N)}\left[\mathbf{h}_{1}\right] \boldsymbol{\Gamma}^{(N+L)}\left[f_{1}-f_{r}\right] \mathbf{T}_{c p}^{(L)} \mathbf{H}_{c p}^{(N)}\left[\mathbf{h}_{1}\right] \mathbf{s}_{1} \\
&+\alpha_{c p} e^{j 2 \pi\left(f_{2}-f_{1}\right) L T_{s}} \boldsymbol{\Gamma}^{(N)}\left[f_{r}-f_{1}\right] \mathbf{H}_{c v}^{(N)}\left[\mathbf{h}_{1}\right] \boldsymbol{\Gamma}^{(N+L)}\left[f_{2}-f_{r}\right] \mathbf{T}_{c p}^{(L)} \mathbf{H}_{c p}^{(N)}\left[\mathbf{h}_{2}\right] \mathbf{s}_{2} \\
&+\underbrace{\alpha_{c p} e^{j 2 \pi\left(f_{r}-f_{1}\right) L T_{s}} \boldsymbol{\Gamma}^{(N)}\left[f_{r}-f_{1}\right] \mathbf{H}_{c v}^{(N)}\left[\mathbf{h}_{1}\right] \mathbf{n}_{r}+\mathbf{n}_{1}}_{\mathbf{n}_{e}}
\end{aligned}
$$

is shown on the top of the next page. The notation $\mathbf{n}_{e}$ denotes the equivalent noise and has the covariance

$$
\begin{aligned}
\mathbf{R}_{n e}=\sigma_{n}^{2} & \left(\alpha_{c p}^{2} \boldsymbol{\Gamma}^{(N)}\left[f_{r}-f_{1}\right] \mathbf{H}_{c v}^{(N)}\left[\mathbf{h}_{1}\right]\right. \\
& \left.\times\left(\mathbf{H}_{c v}^{(N)}\left[\mathbf{h}_{1}\right]\right)^{H}\left(\boldsymbol{\Gamma}^{(N)}\left[f_{r}-f_{1}\right]\right)^{H}+\mathbf{I}\right) .
\end{aligned}
$$

When $N \gg L$, the following approximation can be made:

$$
\mathbf{R}_{n e} \approx \sigma_{n}^{2}\left(\alpha_{c p}^{2} \sum_{l=0}^{L} \sigma_{h_{1, l}}^{2}+1\right) \mathbf{I} .
$$

Before we proceed, let us look at the following lemma:

Lemma 1: The following equality holds for any $\mathbf{H}_{c v}^{(\cdot)}\left[\mathbf{h}_{i}\right]$ and $\boldsymbol{\Gamma}^{(\cdot)}[f]$, where $(\cdot)$ represents the appropriate dimensions:

$$
\mathbf{H}_{c v}^{(N)}\left[\mathbf{h}_{i}\right] \boldsymbol{\Gamma}^{(N+L)}[f]=\boldsymbol{\Gamma}^{(N)}[f] \mathbf{H}_{c v}^{(N)}\left[\boldsymbol{\Omega}^{(L+1)}[f] \mathbf{h}_{i}\right],
$$

where

$$
\boldsymbol{\Omega}^{(K)}[f]=\operatorname{diag}\left\{e^{j 2 \pi f(K-1) T_{s}}, \ldots, e^{j 2 \pi f T_{s}}, 1\right\} .
$$

Proof: Proved from the straightforward computation.

Lemma 1 says that, it is possible to switch $\boldsymbol{\Gamma}^{(\cdot)}[f]$ from the right side of $\mathbf{H}_{c v}^{(\cdot)}\left[\mathbf{h}_{i}\right]$ to the left side by changing the dimension of $\boldsymbol{\Gamma}^{(\cdot)}[f]$ and rotating $\mathbf{h}_{i}$.

According to Lemma $1, \mathbf{y}_{c p}$ in (8) can be rewritten as

$$
\begin{aligned}
\mathbf{y}_{c p}=\alpha_{c p} \mathbf{H}_{c v}^{(N)}\left[\boldsymbol{\Omega}^{(L+1)}\left[f_{1}-f_{r}\right] \mathbf{h}_{1}\right] \mathbf{T}_{c p}^{(L)} \mathbf{H}_{c p}^{(N)}\left[\mathbf{h}_{1}\right] \mathbf{s}_{1} \\
+\alpha_{c p} e^{j 2 \pi v L T_{s}} \boldsymbol{\Gamma}^{(N)}[v] \mathbf{H}_{c v}^{(N)}\left[\boldsymbol{\Omega}^{(L+1)}\left[f_{2}-f_{r}\right] \mathbf{h}_{1}\right] \mathbf{T}_{c p}^{(L)} \\
\times \mathbf{H}_{c p}^{(N)}\left[\mathbf{h}_{2}\right] \mathbf{s}_{2}+\mathbf{n}_{e} \\
=\alpha_{c p} \mathbf{H}_{c p}^{(N)}\left[\boldsymbol{\Omega}^{(L+1)}\left[f_{1}-f_{r}\right] \mathbf{h}_{1}\right] \mathbf{H}_{c p}^{(N)}\left[\mathbf{h}_{1}\right] \mathbf{s}_{1} \\
+\alpha_{c p} e^{j 2 \pi v L T_{s}} \boldsymbol{\Gamma}^{(N)}[v] \mathbf{H}_{c p}^{(N)}\left[\boldsymbol{\Omega}^{(L+1)}\left[f_{2}-f_{r}\right] \mathbf{h}_{1}\right] \\
\times \mathbf{H}_{c p}^{(N)}\left[\mathbf{h}_{2}\right] \mathbf{s}_{2}+\mathbf{n}_{e}
\end{aligned}
$$

where the property (5) is used when deriving the second equality.

We further note that

$$
\mathbf{H}_{c p}^{(N)}\left[\mathbf{x}_{1}\right] \mathbf{H}_{c p}^{(N)}\left[\mathbf{x}_{2}\right]=\mathbf{H}_{c p}^{(N)}\left[\mathbf{x}_{1} \circledast \mathbf{x}_{2}\right],
$$

where $\circledast$ denotes the $N$-point circular convolution between two vectors. Since $N \geq 2 L+1$ is assumed, the non-zero entries in $N$-point circular convolution between $\mathbf{h}_{i}$ 's coincides with the linear convolution of their non-zero entries.
Let $\otimes$ denotes the linear convolution between two vectors. Then $\mathbf{y}_{c p}$ can be expressed as

$$
\begin{aligned}
& \mathbf{y}_{c p}=\alpha_{c p} \mathbf{H}_{c p}^{(N)}\left[(\underbrace{\left.\boldsymbol{\Omega}^{(L+1)}\left[f_{1}-f_{r}\right] \mathbf{h}_{1}\right) \otimes \mathbf{h}_{1}}_{\mathbf{a}_{c p}}] \mathbf{s}_{1}+\mathbf{n}_{e}\right. \\
& +\alpha_{c p} e^{j 2 \pi v L T_{s}} \boldsymbol{\Gamma}^{(N)}[v] \mathbf{H}_{c p}^{(N)}\left[(\underbrace{\left.\boldsymbol{\Omega}^{(L+1)}\left[f_{2}-f_{r}\right] \mathbf{h}_{1}\right) \otimes \mathbf{h}_{2}}_{\mathbf{b}_{c p}}] \mathbf{s}_{2}\right.
\end{aligned}
$$

where $\mathbf{a}_{c p}, \mathbf{b}_{c p}$ are the $(2 L+1) \times 1$ equivalent channel vectors and $v$ is the equivalent $\mathrm{CFO}$, i.e. $v=f_{2}-f_{1}$.

\section{Data detection at terminals}

If the cascaded channel $\mathbf{a}_{c p}$ is known to $\mathbb{T}_{1}$, then the first term on the right-hand side (RHS) of (15) can be removed since $\mathbb{T}_{1}$ knows its own signal $\mathbf{s}_{1}$. If the CFO $v$ is also known, then $e^{j 2 \pi v L T_{s}} \boldsymbol{\Gamma}^{(N)}[v]$ can be compensated and the remaining signal is

$$
\mathbf{z}_{c p}=\alpha_{c p} \mathbf{H}_{c p}^{(N)}\left[\mathbf{b}_{c p}\right] \mathbf{s}_{2}+e^{-j 2 \pi v L T_{s}}\left(\boldsymbol{\Gamma}^{(N)}[v]\right)^{H} \mathbf{n}_{e} .
$$

As long as $\mathbf{b}_{c p}$ is known, the regular OFDM detection can be efficiently performed from fast Fourier Transform (FFT).

\section{E. Joint CFO and channel estimation}

From the above discussion, we know that the task is to estimate $\mathbf{a}_{c p}, \mathbf{b}_{c p}$, and $v$. Assume now $\mathbf{s}_{1}$ and $\mathbf{s}_{2}$ as the training blocks, we can rewrite $\mathbf{y}_{c p}$ as

$$
\mathbf{y}_{c p}=\mathbf{S}_{1}^{(N)} \mathbf{a}_{c p}+\boldsymbol{\Gamma}^{(N)}[v] \mathbf{S}_{2}^{(N)} \mathbf{b}_{c p}+\mathbf{n}_{e}
$$

where $\mathbf{S}_{i}^{(N)}$ is the $N \times(2 L+1)$ circulant matrix with first column $\mathbf{s}_{i}$. Meanwhile, $N \geq 4 L+3$ is required to estimate all the unknown parameters.

\section{Least SQuare Estimation}

For simplification, we can omit the superscript and the subscript of (17) and rewrite it as

$$
\mathbf{y}=\mathbf{S}_{1} \mathbf{a}+\boldsymbol{\Gamma} \mathbf{S}_{2} \mathbf{b}+\mathbf{n}_{e} .
$$

The main difference between this new model and the tractional one [11] is that the CFO component resides only in part of the signal components. 


\section{A. The Algorithm}

The LS estimation can be directly expressed as

$$
\{\widehat{\mathbf{a}}, \widehat{\mathbf{b}}, \widehat{v}\}=\arg \min _{\mathbf{a}, \mathbf{b}, v}\left\|\mathbf{y}-\mathbf{S}_{1} \mathbf{a}-\mathbf{\Gamma} \mathbf{S}_{2} \mathbf{b}\right\|^{2},
$$

where $\boldsymbol{\Gamma}$ stands for $\boldsymbol{\Gamma}[v]$.

Denote $\mathbf{C}=\left[\mathbf{S}_{1}, \boldsymbol{\Gamma} \mathbf{S}_{2}\right]$ and $\mathbf{d}=\left[\mathbf{a}^{T}, \mathbf{b}^{T}\right]^{T}$. When $\mathbf{C}$ is a tall matrix, $\mathbf{d}$ can be obtained as

$$
\widehat{\mathbf{d}}=\left(\mathbf{C}^{H} \mathbf{C}\right)^{-1} \mathbf{C}^{H} \mathbf{y} .
$$

Substituting (20) back to (19), we can estimate CFO as

$$
\begin{aligned}
\widehat{v} & =\arg \min _{v}\|\mathbf{y}-\mathbf{C} \widehat{\mathbf{d}}\|^{2} \\
& =\arg \max _{v} \mathbf{y}^{H} \mathbf{C}\left(\mathbf{C}^{H} \mathbf{C}\right)^{-1} \mathbf{C}^{H} \mathbf{y} \\
& =\arg \max _{v} g(v) .
\end{aligned}
$$

Since there is only one unknown variable, the grid searching method can be immediately applied to find $\widehat{v}$. Moreover, $g(v)$ is a polynomial of $v$, the rooting method suggested in [14] can also be applied to improve the efficiency. Once $\widehat{v}$ is found, the estimates of $\mathbf{a}, \mathbf{b}$ can be obtained from (20).

\section{B. Approximated Cramér-Rao Bound (ACRB)}

In this subsection, we assume the noise covariance as the one in (10) and derive the approximated CRB. Note that, this bound is asymptotically correct for large value of $N$.

Let $\boldsymbol{\mu}=\mathbf{S}_{1} \mathbf{a}+\boldsymbol{\Gamma} \mathbf{S}_{2} \mathbf{b}$, and define

$$
\boldsymbol{\eta} \triangleq\left[v, \Re\{\mathbf{a}\}^{T}, \Im\{\mathbf{a}\}^{T}, \Re\{\mathbf{b}\}^{T}, \Im\{\mathbf{b}\}^{T}\right]^{T} .
$$

Following [13], the Fisher Information Matrix (FIM) is obtained as:

$$
\mathbf{F}=\frac{2}{\sigma_{n e}^{2}} \Re\left[\frac{\partial \boldsymbol{\mu}^{H}}{\partial \boldsymbol{\eta}} \frac{\partial \boldsymbol{\mu}}{\partial \boldsymbol{\eta}^{T}}\right]=\frac{2}{\sigma_{n e}^{2}}\left[\begin{array}{ccc}
F_{11} & \mathbf{r}^{T} & \mathbf{s}^{T} \\
\mathbf{r} & \mathbf{K} & \mathbf{V}^{T} \\
\mathbf{s} & \mathbf{V} & \mathbf{N}
\end{array}\right]
$$

where

$$
\begin{aligned}
F_{11} & =\mathbf{b}^{H} \mathbf{S}_{2}^{H} \mathbf{D}^{2} \mathbf{S}_{2} \mathbf{b}, \\
\mathbf{D} & =2 \pi T_{s} \operatorname{diag}\{0,1, \ldots,(N-1)\} \\
\mathbf{r} & =\left[\begin{array}{c}
-\Im\left(\mathbf{S}_{1}^{H} \mathbf{D} \boldsymbol{\Gamma} \mathbf{S}_{2} \mathbf{b}\right) \\
\Re\left(\mathbf{S}_{1}^{H} \mathbf{D} \boldsymbol{\Gamma} \mathbf{S}_{2} \mathbf{b}\right)
\end{array}\right], \quad \mathbf{s}=\left[\begin{array}{c}
-\Im\left(\mathbf{S}_{2}^{H} \mathbf{D} \mathbf{S}_{2} \mathbf{b}\right) \\
\Re\left(\mathbf{S}_{2}^{H} \mathbf{D} \mathbf{S}_{2} \mathbf{b}\right)
\end{array}\right], \\
\mathbf{K} & =\left[\begin{array}{ll}
\Re\left(\mathbf{S}_{1}^{H} \mathbf{S}_{1}\right) & -\Im\left(\mathbf{S}_{1}^{H} \mathbf{S}_{1}\right) \\
\Im\left(\mathbf{S}_{1}^{H} \mathbf{S}_{1}\right) & \Re\left(\mathbf{S}_{1}^{H} \mathbf{S}_{1}\right)
\end{array}\right] \\
\mathbf{V} & =\left[\begin{array}{ll}
\Re\left(\mathbf{S}_{2}^{H} \boldsymbol{\Gamma}^{H} \mathbf{S}_{1}\right) & -\Im\left(\mathbf{S}_{2}^{H} \mathbf{\Gamma}^{H} \mathbf{S}_{1}\right) \\
\Im\left(\mathbf{S}_{2}^{H} \mathbf{\Gamma}^{H} \mathbf{S}_{1}\right) & \Re\left(\mathbf{S}_{2}^{H} \mathbf{\Gamma}^{H} \mathbf{S}_{1}\right)
\end{array}\right], \\
\mathbf{N} & =\left[\begin{array}{ll}
\Re\left(\mathbf{S}_{2}^{H} \mathbf{S}_{2}\right) & -\Im\left(\mathbf{S}_{2}^{H} \mathbf{S}_{2}\right) \\
\Im\left(\mathbf{S}_{2}^{H} \mathbf{S}_{2}\right) & \Re\left(\mathbf{S}_{2}^{H} \mathbf{S}_{2}\right)
\end{array}\right] .
\end{aligned}
$$

The CRB of CFO is the upper-left block in $\mathbf{F}^{-1}$, which can be explicitly calculated as:

$$
\operatorname{ACRB}(v)=\frac{\sigma_{n e}^{2}}{2}\left[F_{11}-\mathbf{t}_{1}^{T} \mathbf{Q}_{1}^{-1} \mathbf{t}_{1}\right]^{-1},
$$

where

$$
\mathbf{t}_{1}=\left[\begin{array}{l}
\mathbf{r} \\
\mathbf{s}
\end{array}\right], \quad \mathbf{Q}_{1}=\left[\begin{array}{c}
\mathbf{K}, \mathbf{V}^{T} \\
\mathbf{V}, \mathbf{N}
\end{array}\right]
$$

The ACRBs of the channel estimates $\mathbf{a}, \mathbf{b}$ are then given by

$$
\begin{aligned}
& \operatorname{ACRB}(\mathbf{a})=\mathbf{A F}^{-1} \mathbf{A}^{H}, \\
& \operatorname{ACRB}(\mathbf{b})=\mathbf{B F}^{-1} \mathbf{B}^{H},
\end{aligned}
$$

were

$$
\begin{aligned}
& \mathbf{A}=\left[\mathbf{0}_{(2 L+1) \times 1}, \mathbf{I}, j \mathbf{I}, 0 \times \mathbf{I}, 0 \times \mathbf{I}\right] \\
& \mathbf{B}=\left[\mathbf{0}_{(2 L+1) \times 1}, 0 \times \mathbf{I}, 0 \times \mathbf{I}, \mathbf{I}, j \mathbf{I}\right],
\end{aligned}
$$

where I means $(2 L+1) \times(2 L+1)$ identity matrix.

\section{NUMERICAL RESULTS}

A three-tap model for both $\mathbf{h}_{i}$ is assumed, while each tap is Gaussian with unit variance. The variance of the noise is taken as $\sigma_{n}^{2}=1$. The normalized frequencies $f_{1}, f_{r}$, and $f_{2}$ are set as $0.94,1$ and 1.06, respectively. The mean square error (MSE) is chosen as the figure of merit, defined by

$$
\begin{aligned}
& \operatorname{MSE}(v)=\frac{1}{10000} \sum_{i=1}^{10000}\left(\hat{v}_{i}-v\right)^{2}, \\
& \operatorname{MSE}(\mathbf{x})=\frac{1}{10000} \sum_{i=1}^{10000} \frac{1}{3}\left(\hat{\mathbf{x}}_{i}-\mathbf{x}\right)^{2},
\end{aligned}
$$

where $\mathbf{x}$ represents $\mathbf{a}$ or $\mathbf{b}$, and 10000 is the number of the Monte-Carlo trials used for average.

First we examine the performance of CFO estimation and the corresponding MSEs versus SNR curves are shown in Fig. 2 for $N=16$ and $N=32$, respectively. The CRBs are also displayed for comparison. It is seen that for both values of $N$, CFO estimation MSEs approach their bounds in high SNR region. The mismatch at the low SNR region is generally known as outlier [14], that happens because of the estimation ambiguity in several Monte-Carlo runs, which ruins the average performance.

We then demonstrate the corresponding channel estimation results, as well as the CRBs in Fig.3. We see that the

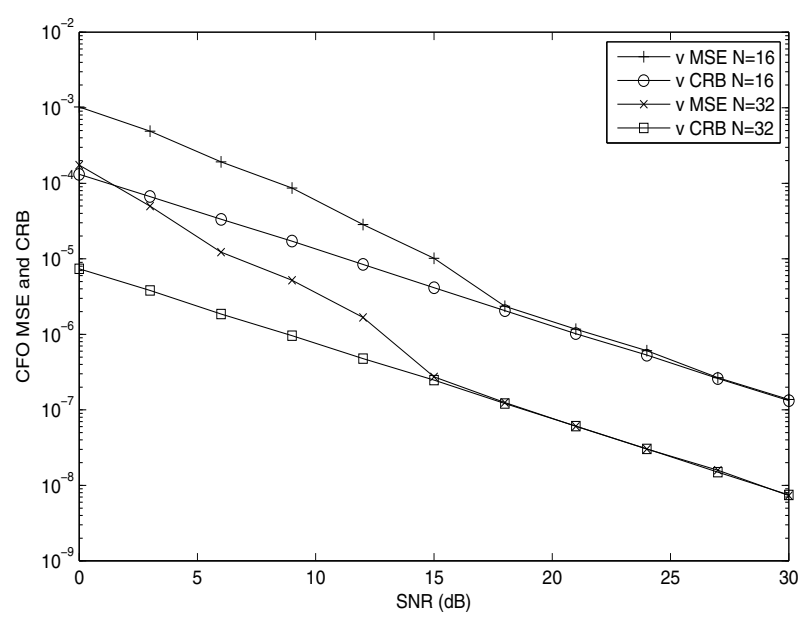

Fig. 2. CFO estimation MSEs versus SNR for $N=16,32$, respectively. 


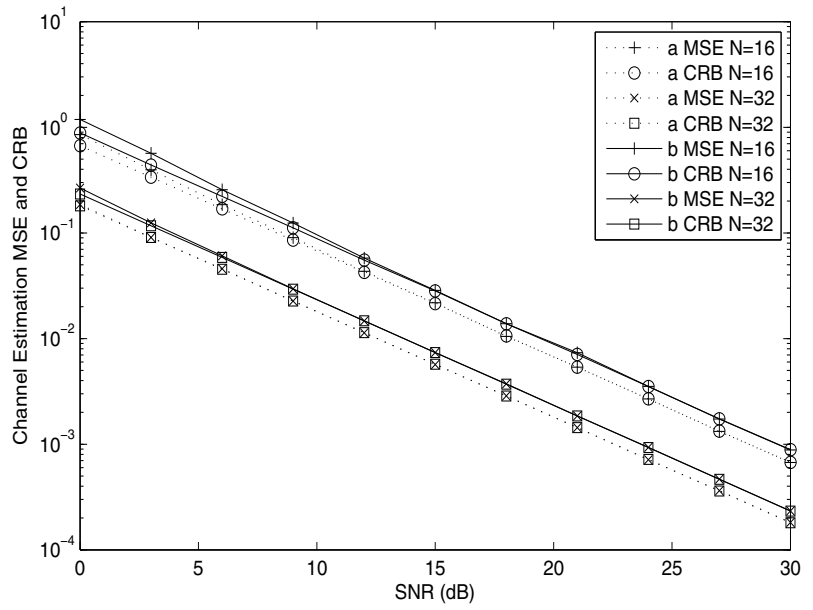

Fig. 3. Channel estimation MSEs versus SNR for $N=16$, 32, respectively.

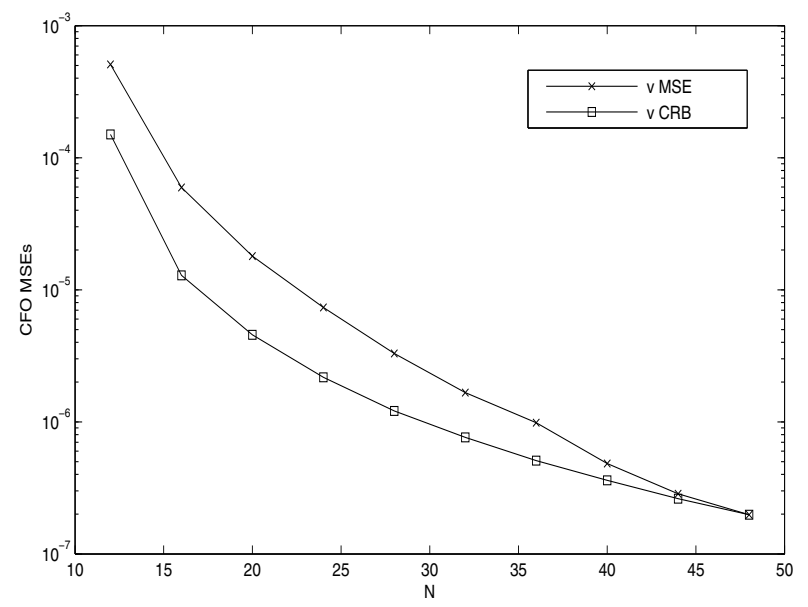

Fig. 4. CFO estimation MSEs versus $N$ for $\mathrm{SNR}=10 \mathrm{~dB}$.

estimation MSEs approach their corresponding bounds much faster than CFO estimation. This is because the errors in the estimated phase have less effect on the channel estimation but have severe effect on the CFO estimation. However, when CFO errors are too large at low SNR region, the channel estimation results still deviate from its theoretical values.

Next we set $\mathrm{SNR}=10 \mathrm{~dB}$ and examine the estimation performance versus the block length $N$. The corresponding simulation results are shown in Fig. 4 and Fig. 5, respectively. It is seen that with the increasing of $N$, the outlier effect in CFO estimation diminishes. Moreover, the performance of both CFO and channel estimation improves when $N$ gets larger.

\section{CONCLUSiOnS}

In this paper, we considered the problem of joint $\mathrm{CFO}$ and channel estimation for AF TWRN in a frequency-selective environment. We adapted the traditional CP OFDM modulation to TWRN that is able to realize the multi-carrier transmission as well as facilitate the system initialization. We then

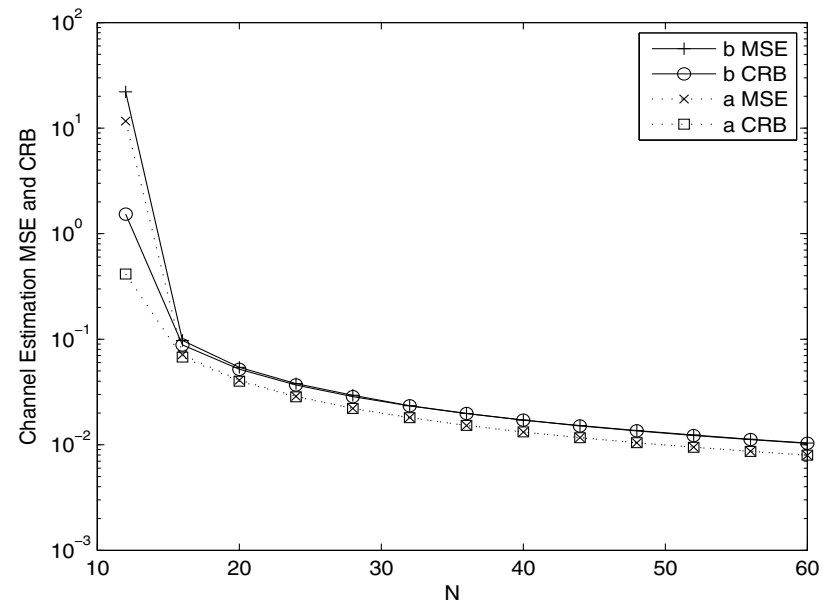

Fig. 5. Channel estimation MSEs versus $N$ for $\mathrm{SNR}=10 \mathrm{~dB}$.

proposed a joint LS estimator and derived its corresponding approximated CRBs. These bounds are asymptotically precise for large $N$. Finally, the numerical results verified the proposed studies.

\section{REFERENCES}

[1] J. N. Laneman, D. N. C. Tse, and G. W. Wornell, "Cooperative diversity in wireless networks: efficient protocols and outage behavior," IEEE Trans. Inform. Theory, vol. 50, no. 12, pp. 3062-3080, Dec. 2004.

[2] S. G. S. Katti and D. Katabi, "Embracing wireless interference: analog network coding," in Computer Science and Artificial Intelligence Laboratory Technical Report, Feb. 2007.

[3] B. Rankov and A. Wittneben, "Spectral efficient signaling for halfduplex relay channels," in Proc. Annual Conference on Signals, Systems, and Computers, Pacific Grove, USA, Oct. 2005, pp. 1066-1071.

[4] B. Rankov and A. Wittneben, "Achievable rate regions for the two-way relay channel," in Proc. IEEE ISIT, Seattle, USA, July 2006, pp. 16681672.

[5] S. J. Kim, N. Devroye, P. Mitran, and V. Tarokh, "Achievable rate regions for bi-directional relaying," submitted to IEEE Trans. Inform. Theory, avalaible at arXiv:0808.0954v1.

[6] T. Cui, T. Ho, and J. Kliewer, "Memoryless relay strategies for two-way relay channels: Performance analysis and optimization," in Proc. IEEE ICC, Beijing, China, May 2008, pp. 1139-1143.

[7] T. Cui, F. Gao, T. Ho, and A. Nallanathan, "Distributed space-time coding for two-way wireless relay networks," in Proc. IEEE ICC, Beijing, China, May 2008, pp. 3888-3892.

[8] R. Zhang, Y.-C. Liang, C. C. Chai, and S. G. Cui, "Optimal beamforming for two-way multi-antenna relay channel with analogue network coding," IEEE J. Select. Areas Commun., vol. 27, no. 5, pp. 699-712, June 2009.

[9] C. K. Ho, R. Zhang and Y.-C. Liang, "Two-way relaying over OFDM: optimized tone permutation and power allocation," in Proc. IEEE ICC, Beijing, China, May 2008, pp. 3908-3912.

[10] G. Wang, F. Gao, and C. Tellambura, "Joint frequency offset and channel estimation methods for two-way relay networks," to appear in Proc. IEEE GLOBECOM, Honolulu, Hawaii, Nov./Dec. 2009.

[11] M. Morelli and U. Mengali, "Carrier-frequency estimation for transmissions over selective channels," IEEE Trans. Commun., vol. 48, no. 9, pp. 1580-1589, Sep 2000.

[12] F. Gao, R. Zhang, and Y.-C. Liang, "Channel estimation for ofdm modulated two-way relay networks," IEEE Trans. Signal Processing, vol. 57, no. 11, pp. 4443-4455, Nov. 2009.

[13] P. Stoica and O. Besson, "Training sequence design for frequency offset and frequency-selective channel estimation," IEEE Trans. Commun., vol. 51, no. 11, pp. 1910-1917, Nov. 2003.

[14] F. Gao and A. Nallanathan, "Blind maximum likelihood cfo estimation for ofdm systems via polynomial rooting," IEEE Signal Processing Lett., vol. 13, no. 2, pp. 73-76, Feb. 2006. 